\title{
Dual topology of functional Toll-like receptor 3 expression in human hepatocellular carcinoma: Differential signaling mechanisms of TLR3-induced NF- $\mathrm{kB}$ activation and apoptosis
}

\author{
KENTARO YONEDA ${ }^{1}$, KAZUSHI SUGIMOTO $^{1}$, KATSUYA SHIRAKI ${ }^{1}$, JUNICHIRO TANAKA ${ }^{1}$, \\ TETSUYA BEPPU ${ }^{1}$, HIROYUKI FUKE ${ }^{1}$, NORIHIKO YAMAMOTO ${ }^{1}$, MASAHIRO MASUYA ${ }^{2}$, \\ RYO HORIE $^{3}$, KAZUHIKO UCHIDA ${ }^{3}$ and YOSHIYUKI TAKEI ${ }^{1}$
}

\author{
${ }^{1}$ Department of Gastroenterology, Mie University Graduate School of Medicine, 2-174 Edobashi, Tsu, Mie 514-8507; \\ ${ }^{2}$ The Blood Transfusion Service, Mie University Hospital, Tsu, Mie 514-0001; ${ }^{3}$ Graduate Schoool of \\ Comprehensive Human Sciences, University of Tsukuba, 1-1-1 Tennoudai, Tsukuba, Ibaraki 305-0006, Japan
}

Received March 28, 2008; Accepted May 19, 2008

DOI: 10.3892/ijo_00000080

\begin{abstract}
Toll-like receptor 3 (TLR3) is a pattern-recognizing receptor that is involved in immune signaling and plays a crucial role in survival by being able to recognize various viral components including double-stranded RNA (dsRNA). TLR3 expression and function in cancer cells are not well understood. We investigated the expression of TLR3 in hepatocellular carcinoma (HCC) cells and the function of TLR3 signaling by stimulation and transfection with polyinosinic-polycytidylic acid (Poly I:C), a synthetic form of dsRNA. TLR3 mRNA was expressed in HCC tissues as well as in non-tumor tissues. Positive immunohistochemical staining for TLR3 was observed in $52.7 \%$ of HCC tissues, and in HCC cells we found both membranous and cytoplasmic expression of TLR3. While cell surface stimulation of TLR3 with Poly I:C did not affect
\end{abstract}

Correspondence to: Dr Katsuya Shiraki, Department of Gastroenterology, Mie University Graduate School of Medicine, 2-174 Edobashi, Tsu, Mie 514-8507, Japan

E-mail: katsuyas@clin.medic.mie-u.ac.jp

Abbreviations: TLR3, Toll-like receptor 3; dsRNA, double-strand RNA; HCC, hepatocellular carcinomas; Poly I:C, polyinosinicpolycytidylic acid; NF- $\kappa B$, nuclear factor $\kappa B$; TRAIL, TNF-related apoptosis-inducing ligand; TRIF, Toll-IL1 receptor-resistance domain-containing adaptor inducing IFN- $\beta$; $\mathrm{HBV}$, hepatitis B virus; $\mathrm{HCV}$, hepatitis C virus; ssRNA, single-stranded RNA; LPS, lipopolysaccharide; Lipo, lipofectamine 2000; CHX, cycloheximide; Act-D, actinomycin D; DAB, 3,3'-diaminobenzidene-hydrogen peroxidase; MTT, 3-(4,5-dimethylthiazol-2-yl)-2,5-diphenyl tetrazolium bromide assay; DAPI, 4'6,-diamidino-2-phenylindole; FLIP, FLICE/caspase 8 inhibitory protein; XIAP, X-linked inhibitor of apoptosis proteins

Key words: Toll-like receptor 3, hepatocellular carcinoma, nuclear factor $\mathrm{\kappa B}$, apoptosis cell viability, it did activate NF- $\mathrm{BB}$ levels. In contrast, cytoplasmic stimulation with transfected Poly I:C significantly induced apoptosis accompanied by the down-regulation of anti-apoptotic protein. Transfected Poly I:C also synergistically augmented TRAIL-induced apoptosis, but only with low levels of transfected Poly I:C was IFN- $\beta$ production not observed. In conclusion, our results indicate that TLR3 expression in HCC plays an important role with regard to cell survival and proapoptotic activity. Endogenously expressed TLR3 may provide new clinical prospects for TLR3 agonists as cytotoxic agents in HCC.

\section{Introduction}

Toll-like receptors (TLRs) are involved in the innate immunity against microbial pathogens such as bacteria, protozoa, fungi or viruses. These receptors are type I transmembrane proteins and play a critical role in the subsequent induction of adaptive immune responses (1-5). Stimulation of TLRs can induce a range of innate and adaptive immune responses through cytokines, interferons, chemokines and cell surface molecules, as well as increase effector functions.

TLRs are broadly distributed in various cells of the immune system, including polymorphonuclear phagocytes, monocytes, dendritic cells and natural killer cells, as well as some epithelial and endothelial cells (6-9). However, to date, the specific subcellular localization of TLRs remains to be determined. TLR 1, 2, 4, 5 and 6 are present in the plasma membrane and TLR3, 7, 8 and 9 are present in endosomes and most likely are involved in signal pathways (10). Via the TLR signaling pathways, the liver is continuously exposed to a large variety of antigens, such as dietary antigens, bacterial toxins and several proinflammatory cytokines through TLR signaling (11). Hepatocytes express low levels of TLR2 and 4 and are responsive to lipopolysaccharide (LPS), and Kupffer cells as well as hepatic stellate cells, biliary epithelial cells and sinusoidal endothelial cells, which express TLR4, produce several proinflammatory cytokines in response to LPS (11-13). However, the exact mechanism responsible for the expression 
and function of TLR in liver diseases has yet to be fully elucidated.

Adapter proteins are recruited to specific TLR intracellular domains and dictate the signaling pathway activations that subsequently are responsible for resistance to pathogens or cell death. Four types of molecules are known, and MyD88 is involved in the majority of TLR pathways, except for TLR3 and 8. TLR3 signaling depends solely on the TIR domain which contains the adaptor-inducing IFN- $\beta$ (TRIF) adapter protein. This leads to activation of the NF- $\kappa$ B and IRF3 transcription factors, which induce the antiviral interferon response $(14,15)$. Furthermore, TRIF itself exhibits proapoptotic activity, suggesting that TLR3 signaling can lead to cell death (16). Hepatitis B virus (HBV) and hepatitis C virus (HCV) induce the type I IFN response, which influences the pathogenesis of chronic viral hepatitis. NS3/4A proteases in $\mathrm{HCV}$ disrupt TRIF, thereby blocking TBK1 (17-20). This suggests that the TLR3 pathway plays an important role in the pathogenesis of HBV and HCV infections.

Recently, the TLR3 ligand dsRNA has been reported to induce apoptosis in several cell types through multiple pathways. In addition, TLR3 may directly trigger apoptosis in certain cancer cells $(21,22)$. Previous studies suggest that the neoplastic process may sabotage the TLR signaling pathways, thereby allowing for unhindered progression of the cancer. In a murine metastasis cancer model, LPS induced tumor metastasis by promoting increased angiogenesis and tumor cell invasion (23). Furthermore, TLRs on tumor cells facilitate their evasion from immune surveillance via the suppression of $\mathrm{T}$-cell proliferation and natural killer cell activity. This suggests that TLR signaling in tumor cells is associated with the progression of cancer and the evasion of host defenses. However, little is known regarding the significance of TLR3 in human carcinoma cells, including hepatocellular carcinoma (HCC) cells.

Therefore, we investigated the expression of TLR3 in HCC cells and tissues by examining the function of the TLR3 signaling which occured after stimulation and transfection with polyinosinic-polycytidylic acid (Poly I:C).

\section{Materials and methods}

Cells and HCC tissues. Human HCC cells HepG2 (JCRB 1054), HLE (JCRB 0404), Huh7 (JCRB0403) and PLC/ $\mathrm{PRF} / 5$ (JCRB0406) were purchased from the Human Science Research Resource Bank (Osaka, Japan). The HCC cells SK-Hep1 and colonic adenocarcinoma cells Colo 320 were purchased from the American Type Culture Collection (Rockville, MD). All cells were cultured in DMEM at $37^{\circ} \mathrm{C}$, supplemented with $1 \%$ penicillin/streptomycin (Gibco BRL, Grand Island, NY) and 10\% heat-inactivated fetal calf serum (Gibco BRL).

A total of 74 HCC tissues (9 non-tumor tissues which included 4 from patients with cirrhotic liver and 5 from patients with chronic hepatitis, and 23 tissues with metastasis from HCC tissues) were obtained from tissue array slides (SuperBioChips Laboratories, Seoul, Korea). We obtained informed consent from all patients prior to the subsequent use of their resected tissues. Resected tissues were frozen immediately at $-80^{\circ} \mathrm{C}$ or fixed in $10 \%$ formalin.
Reagents. Poly I:C, cycloheximide (CHX) and actinomycin (Act-D) were obtained from Sigma (St. Louis, MO), and Lipofectamine 2000 (Lipo) was obtained from Invitrogen (Carlsbad, CA).

Profiling analysis of TLRs recognized by viral components. Total RNA from 16 patients was extracted using Trizol (Life Technologies, Rockville, MD). RNA concentrations and purity were determined using the NanoDrop ND-1000 spectrophotometer (NanoDrop, Rockland, DE) and 2100 Bioanalyser (Agilent, Palo Alto, CA), respectively. Synthesis of biotinlabeled cDNA probes, and hybridization, washing, staining and scanning steps were performed according to the manufacturer's instructions (Affymetrix Inc, Santa Clara, CA).

Flow cytometric analysis. Non-permeabilized live HCC cell lines and colonic adenocarcinoma cells were cultured for $24 \mathrm{~h}$, washed with PBS, and incubated with PE-anti-TLR3 antibody (Santa Cruz Biotechnology, Santa Cruz, CA) and control IgG1. Cells were analyzed with FACScan using CellQuest software (Becton Dickinson, Tokyo, Japan).

Immunohistochemical staining. Immunohistochemical staining for TLR3 was performed on HCC tissues and nonHCC tissues using the labeled streptavidin-biotin method. Deparaffinized sections were heated for $5 \mathrm{~min}$ at $120^{\circ} \mathrm{C}$ in a pressure cooker to reactivate the antigen. Sections were blocked and covered with anti-TLR3 antibody overnight at $4^{\circ} \mathrm{C}$. Sections were incubated with a second biotinylated antibody, followed by avidin-biotin-peroxidase complex. Sections were then developed in a substrate solution of $0.01 \%$ 3,3'-diaminobenzidene-hydrogen peroxidase and counterstained with $10 \%$ hematoxylin.

Detection of TLR3 by immunofluorescence. A total of $2 \times 10^{4}$ cells were cultured in an 8 -well Lab-tek II chamber slide (Nunc $^{\text {TM }}$ Brand Products, Denmark) for $24 \mathrm{~h}$. Live cells were incubated for $1 \mathrm{~h}$ at $4{ }^{\circ} \mathrm{C}$ with FITC-anti-TLR3 antibody, and then visualized after fixation with $2 \%$ paraformaldehyde. Similar experiments were performed with permeabilized cells fixed with $2 \%$ paraformaldehyde followed by treatment with $0.1 \%$ Saponin.

Detection of cell viability and apoptosis assays. To assess the viability of HCC cells, a 3-(4,5-dimethylthiazol-2-yl)-2,5diphenyl tetrazolium bromide (MTT) assay was performed with a Cell Titer 96 assay kit (Promega, Madison, WI). A total of $2 \times 10^{4} \mathrm{HCC}$ cells were cultured in a chamber slide for $24 \mathrm{~h}$ followed by addition of $1 \mu \mathrm{g} / \mathrm{ml}$ Poly I:C or Poly I:C with Lipo. After incubation for $24 \mathrm{~h}$, cell nuclei were stained with 4'6,-diamidino-2-phenylindole (DAPI; Sigma).

Detection of apoptosis-related proteins by immunoblotting. Expression of FLIP, Bcl-xL, XIAP, survivin and caspase 3 in HCC cell lines was analyzed by immunoblotting. Briefly, cells were harvested after stimulation with Poly I:C and Lipo $(0-10 \mu \mathrm{g} / \mathrm{ml})$. Cells were then lysed on ice in lysis buffer (50 mM Tris- $\mathrm{HCl}$ at $\mathrm{pH} 8.0,150 \mathrm{mM} \mathrm{NaCl}, 5 \mathrm{mM}$ ethylenediaminetetraacetic acid, 1\% NP40 and $1 \mathrm{mM}$ PMSF). Equal amounts of extracted proteins were separated by SDS-PAGE 
A

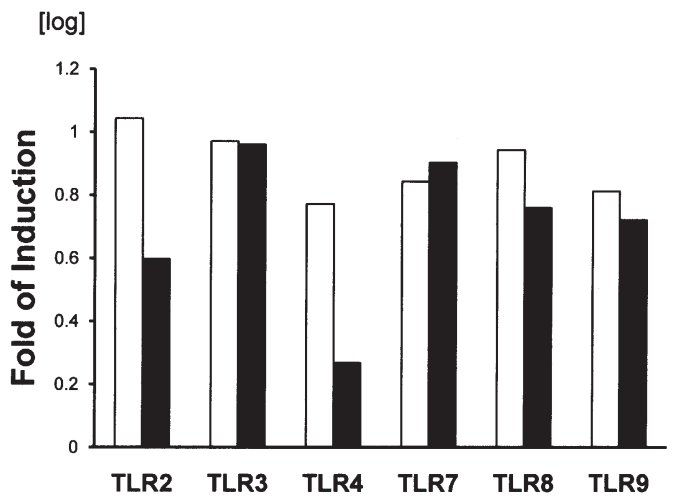

B

\section{TLR3}

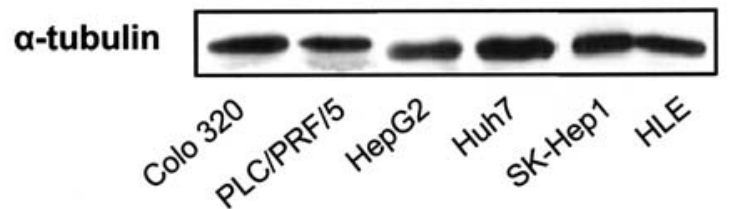

C

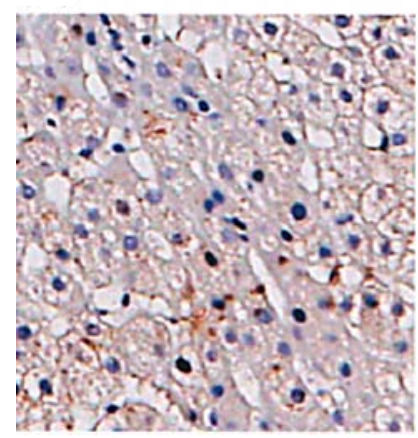

D

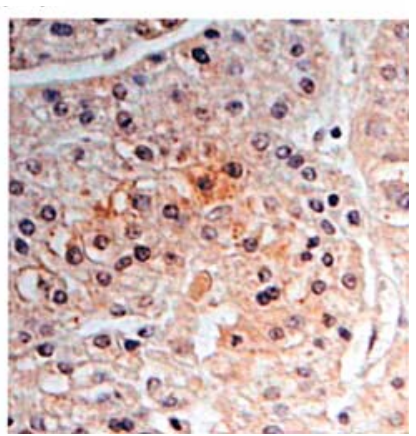

Figure 1. (A) Gene expression of TLRs in 16 normal tissues (open columns) and 16 tumor tissues (closed columns) was analyzed. Of the TLRs, six recognized viral structural components. The $\mathrm{Y}$-axis indicates normalized expression values. (B) Western blot analysis of TLR3 in colonic adenocarcinoma cells (Colo 320) and human HCC cells (PLC/PRF/5, HepG2, Huh7, SK-Hep1 and HLE). Cell lysates were separated by SDS-PAGE and were transferred to nitrocellulose. (C and D) Expression of TLR3 in both non-HCC and HCC. Immunohistochemical staining for TLR3 in a human non-HCC specimen (C) and immunohistochemical staining for TLR3 in a human HCC specimen (D). Note TLR3 staining not only in the cytoplasm, but also in the membrane (original magnification, $\mathrm{x} 100$ ).

and transferred onto nitrocellulose membranes. Blots were probed overnight at $4^{\circ} \mathrm{C}$ with anti-survivin antibody (Santa Cruz Biotechnology), anti-FLIP antibody (MBL, Nagoya, Japan), anti-BCL-xL antibody (Transduction; Lexington, KY), mouse anti-caspase 3 antibody (MBL), anti-XIAP antibody (Transduction) or anti- $\alpha$-tubulin antibody (Oncogene Research Products, San Diego, CA).

$N F-\kappa B$ activity assays and measurement of IFN- $\beta$. The NF- $\mathrm{B}$ activity assay was performed using a Transfactor NF- $\kappa \mathrm{B}$ p65 Colorimetric Kit (Clontech Laboratories, Takara Bio Inc.) according to the manufacturer's instructions.
Table I. Expression of TLR3 in HCCs and non-tumor tissues.

\begin{tabular}{lrrr}
\hline & \multicolumn{3}{c}{ Staining } \\
\cline { 2 - 4 } Histology & None/weak & \multicolumn{1}{c}{ Mild } & Strong \\
\hline Non-tumor tissues & $2(22.3 \%)$ & $4(44.4 \%)$ & $3(33.3 \%)$ \\
HCC & & & \\
Poorly differentiated & $10(55.6 \%)$ & $6(33.3 \%)$ & $2(11.1 \%)$ \\
Moderately differentiated & $18(42.9 \%)$ & $15(35.7 \%)$ & $9(21.4 \%)$ \\
Well differentiated & $7(50.0 \%)$ & $6(42.9 \%)$ & $1(7.1 \%)$ \\
Metastasis from HCC & $15(65.2 \%)$ & $5(21.7 \%)$ & $3(13.1 \%)$ \\
\hline
\end{tabular}

The amount of secreted IFN- $\beta$ in the culture medium of HCC cells was measured using a human IFN-B ELISA Kit (PBL Biomedical Laboratories, Piscataway, NJ).

\section{Results}

TLR3 is expressed in HCC cells and liver tissues. First, we examined cDNA levels of TLRs recognizing viral structural components. Using a cDNA chip system, in tumor and normal tissues, we confirmed that TLR2, 4, 8 and 9 were downregulated in tumors, as compared with normal tissues, although expression of TLR3 and 7 in tumor tissues was almost equal to that in normal tissues (Fig. 1A). Therefore, we focused on TLR3 which recognizes dsRNA.

TLR3 protein expression levels were analyzed by Western blot analysis in cell lysates of cancer cells. As shown in Fig. 1B, TLR3 was detected in all HCC cells and in colonic adenocarcinoma cells, indicating a high prevalence of TLR3 expression in human HCC.

TLR3 expression was investigated in the liver tissues by using immunohistochemical staining in both non-HCC and HCC lesions (Fig. 1C and D). While in 39 of 74 HCC cases $(52.7 \%)$ there was clear TLR3 positivity, no differences were observed for the histological grades. TLR3 staining was not only present in the cytoplasm, but was also present in the membrane. There was also little difference noted in the TLR3 staining patterns between the non-tumor and tumor tissues. In metastasis from HCC, 8 of 23 cases (34.8\%) showed TLR3 positivity (Table I).

TLR3 is expressed on the cell surface in HCC cells. Since receptor localization depends upon the cell type, we used flow cytometric analysis to investigate the cellular localization of TLR3 in HCC cells. As shown in Fig. 2A, significant detectable surface staining for TLR3 was observed in both the HCC and colonic adenocarcinoma cells. To determine the orientation of the TLR3 protein which was expressed on the cell surface of the HCC cells, nonpermeabilized live HepG2 cells were stained with FITC-labeled TLR3 antibody (Fig. 2B and C). Staining was found to be particularly strong in the outer lining of these nonpermeabilized cells, indicating there was localization of TLR3 on the cell surface. In contrast, there was staining of permeabilized cells in both the outer cell lining and cytoplasm. 
A

\section{Colo 320}

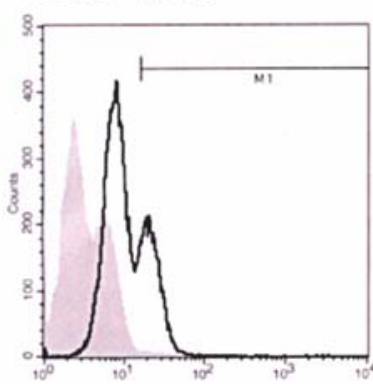

HepG2

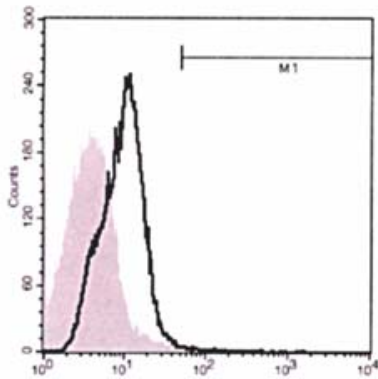

SK-Hep1

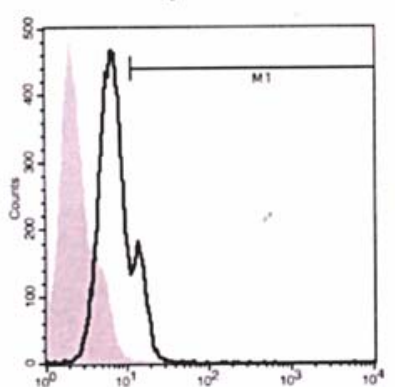

$\mathbf{B}$
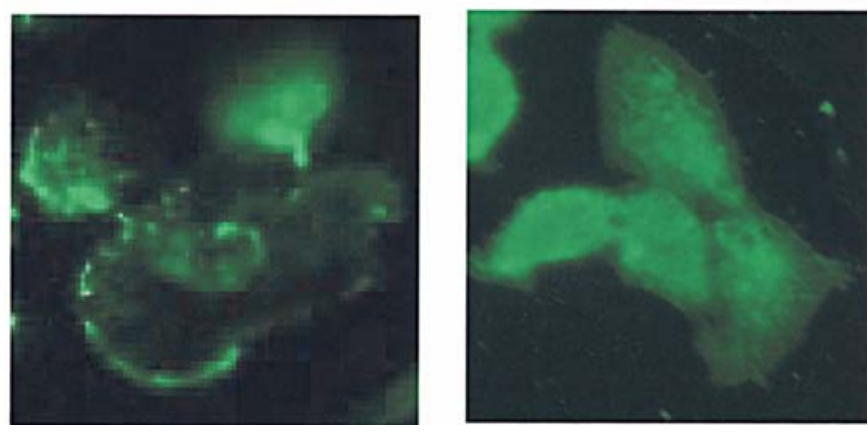

Figure 2. (A) Cell surface expression of TLR3 in colonic adenocarcinoma and HCC cells. Colonic adenocarcinoma and HCC cells were incubated for $48 \mathrm{~h}$ and stained using PE-anti-TLR3. Autofluorescence was determined using cells incubated with PE-control mouse IgG (gray columns). (B and C) Expression of TLR3 in HCC cells. Immunofluorescence staining of live HepG2 cells with TLR3 antibody (B) (x400), and immunofluorescence staining of permeabilized HepG2 cells when using Saponin (C) (x400).

Poly I:C did not affect cell proliferation but induced $N F-\kappa B$ activation. In order to determine the biological significance of the signaling that occurrs via the cell surface TLR3 in HCC cells, we investigated the cytotoxicity of Poly I:C. As shown
A
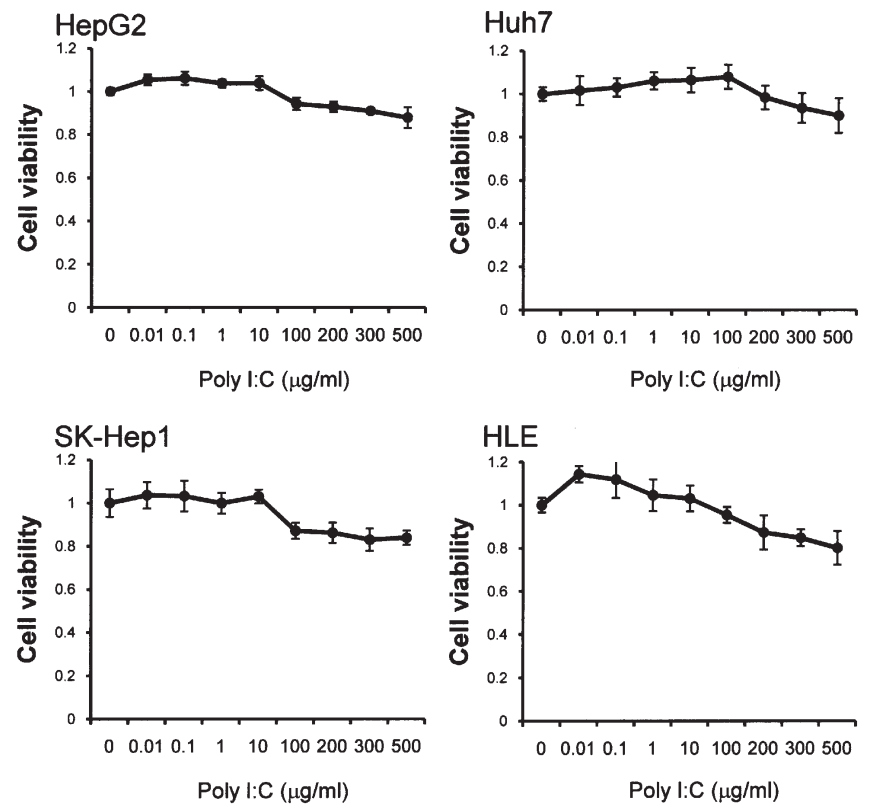

B

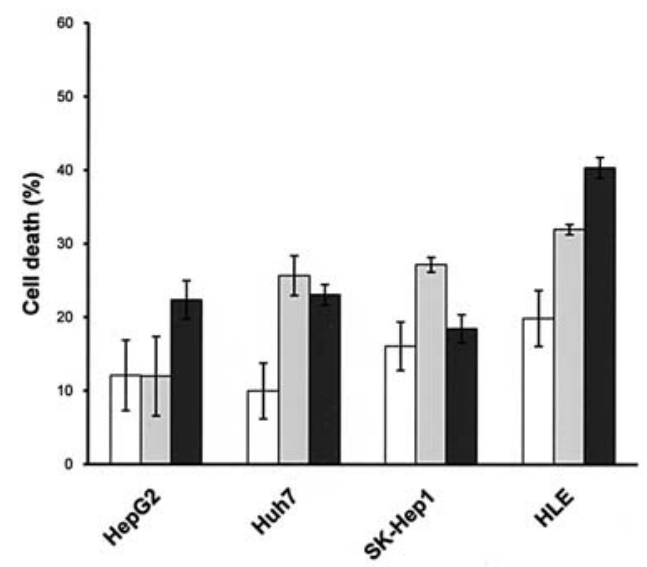

C

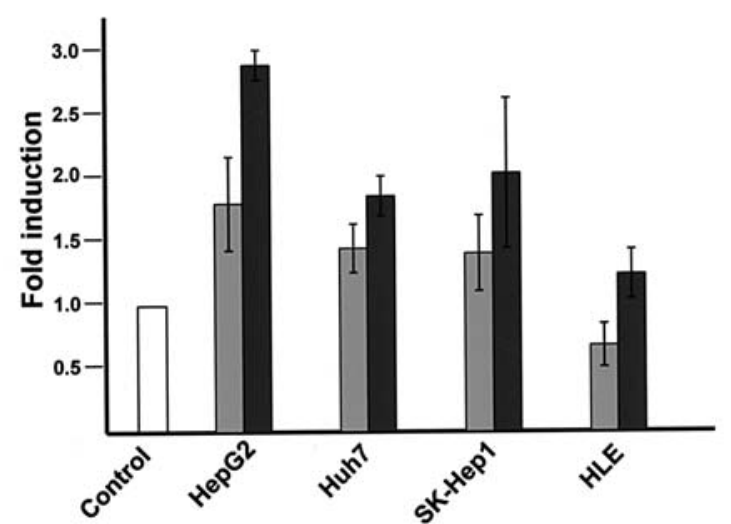

Figure 3. (A) Effects of Poly I:C on HCC cells. HCC cells were incubated with various concentrations of Poly I:C for $48 \mathrm{~h}$. Cell viability was assessed using the MTT assay. Data shown are the means \pm SD of six independent experiments. (B) Cell death in HCC cells incubated with $500 \mu \mathrm{g} / \mathrm{ml}$ Poly I:C alone (open columns), with $500 \mu \mathrm{g} / \mathrm{ml}$ Poly I:C and $0.5 \mu \mathrm{g} / \mathrm{ml}$ actinomycin D (gray columns), or with $500 \mu \mathrm{g} / \mathrm{ml}$ Poly I:C and $4 \mu \mathrm{g} / \mathrm{ml}$ cycloheximide (closed columns). Cell death was assessed by MTT assay. Data shown are the means \pm SD of six independent experiments. (C) Effects of Poly I:C on $\mathrm{NF}-\mathrm{kB}$ activation in HCC cells. HCC cells were treated with $1 \mu \mathrm{g} / \mathrm{ml}$ (gray columns) or $10 \mu \mathrm{g} / \mathrm{ml}$ of Poly I:C (closed columns) for $48 \mathrm{~h}$. NF-kB activation was assessed by ELISA. Data shown are the means \pm SE of three independent experiments. 
A
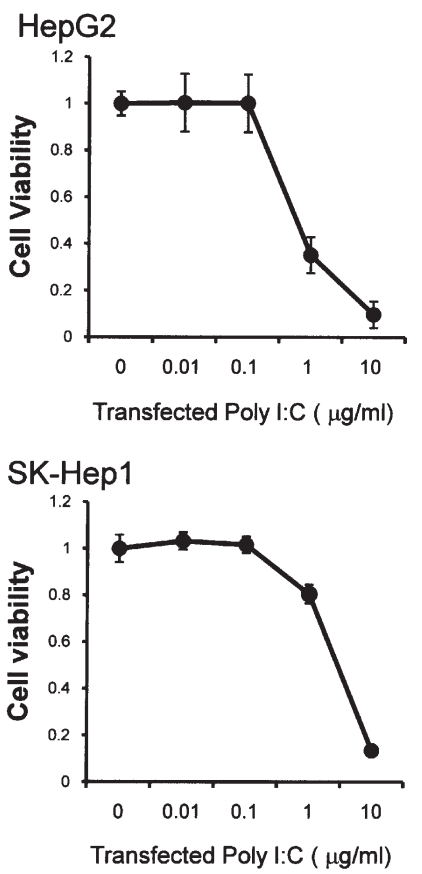

B

Control
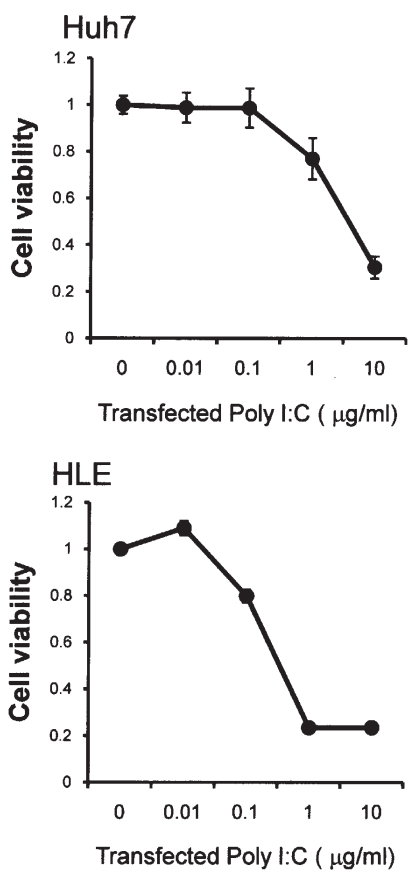

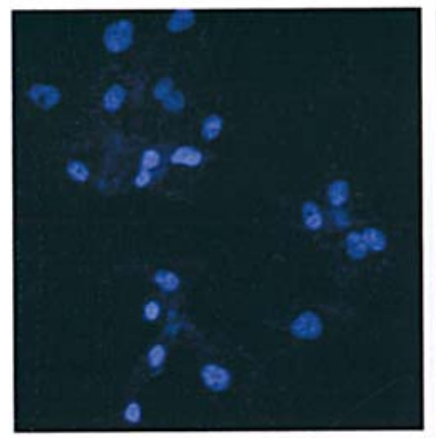

Lipo

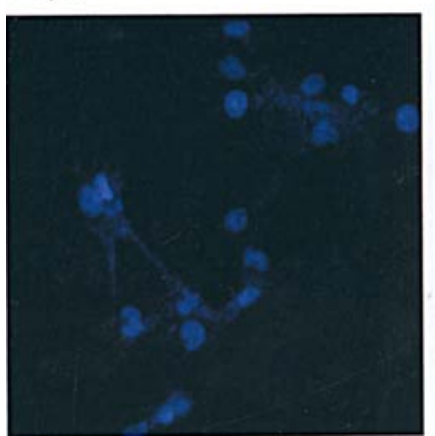

Poly I:C

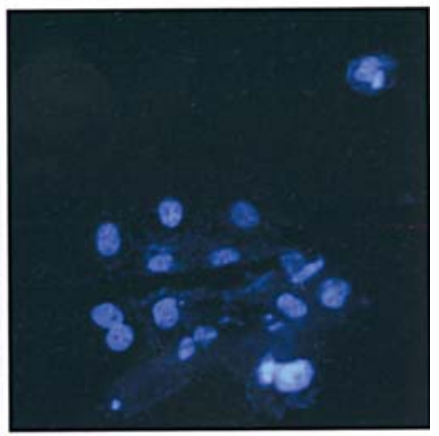

Transfected Poly I:C

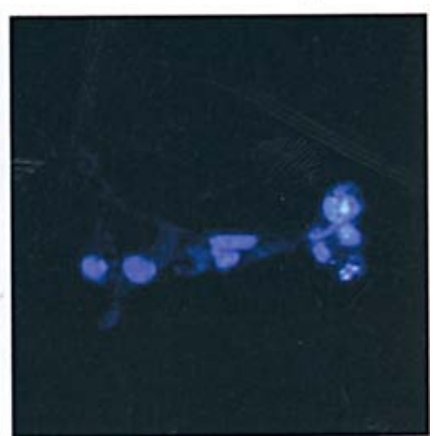

Figure 4. (A) Effects of transfected Poly I:C on HCC cells. HCC cells were incubated with various concentrations of transfected Poly I:C for 48 h. Cell viability was assessed using the MTT assay. Data shown are the means \pm SD of six independent experiments. (B) Transfected Poly I:C-induced apoptosis in Huh7 cells. Cells were incubated with $1 \mu \mathrm{g} / \mathrm{ml}$ Poly I:C, $2.5 \mu \mathrm{l} / \mathrm{ml}$ Lipo and $1 \mu \mathrm{g} / \mathrm{ml}$ Poly I:C $+2.5 \mu 1 / \mathrm{ml}$ Lipo for $24 \mathrm{~h}$. Cell nuclei were then visualized by DAPI staining.

in Fig. 3A, Poly I:C slightly decreased the cell viability in HCC cells.

Subsequently, we examined the effects of the RNAsynthesis inhibitor, Act-D, or the protein synthesis inhibitor, CHX, on Poly I:C-induced cell death (Fig. 3B). The combination of $500 \mu \mathrm{g} / \mathrm{ml}$ Poly I:C and subtoxic levels of the metabolic inhibitors resulted in cell death, particularly in the Huh7 and HLE cells. These results suggest that endogenous suppressors of Poly I:C-mediated apoptosis might be present in HCC cells.

We then examined NF- $\mathrm{BB}$ activation in $\mathrm{HCC}$ cells. In all of the HCC cells, Poly I:C induced a 2- to 2.5-fold activation of NF- $\mathrm{BB}$ activity in a dose-dependent manner (Fig. 3C).

Transfected Poly I:C-induced apoptosis in HCC cells. Since TLR3 was also present in the cytoplasm in addition to its location on the cell surface, we attempted to investigate the cytotoxicity of transfected Poly I:C in HCC cells after stimulating cellular TLR3 by transfection with Poly I:C. Surprisingly, we found that stimulation of transfected Poly I:C for $48 \mathrm{~h}$ resulted in decreased cell viability in a dose-dependent manner (Fig. 4A).

We then performed DAPI nuclear staining in order to determine whether apoptosis occurred. While apoptosis was not induced in cells with PolyI:C by itself, when HCC cells were treated with transfected PolyI:C, typical apoptotic features were observed (Fig. 4B).
Transfected Poly I:C augmented tumor necrosis factor-related apoptosis-inducing ligand (TRAIL)-induced apoptosis in HCC cell lines. Since most HCC cells are resistant to TRAIL, we investigated the effects of TLR3 signaling on TRAIL-induced apoptosis (24). We incubated HCC cells with different concentrations of TRAIL and Poly I:C or transfected Poly I:C for $24 \mathrm{~h}$. Although $1 \mu \mathrm{g} / \mathrm{ml}$ Poly I:C and TRAIL did not affect cell viability in Huh7 cells, stimulation performed with $1 \mu \mathrm{g} / \mathrm{ml}$ transfected Poly I:C augmented the TRAILinduced apoptosis in a dose-dependent manner (Fig. 5A and B).

To investigate the molecular mechanisms of the noted increased apoptotic sensitivity which occurred when using transfected Poly I:C, we used immunoblotting to analyze the expression of the apoptosis-related protein levels (Fig. 5C). We found that cleaved caspase 3 levels were dose-dependent, $1 \mu \mathrm{g} / \mathrm{ml}$ transfected Poly I:C decreased the expression levels of Bcl-xL and survivin, and $10 \mu \mathrm{g} / \mathrm{ml}$ transfected Poly I:C decreased the expression levels of FLIP and XIAP.

Transfected Poly I:C did not activate the IFN- $\beta$ secretion pathway. Finally, we examined TLR3-mediated induction of IFN-B. We used immunoblotting to determine the expression levels of the interferon regulatory factor 3 (IRF-3) and phosphorylated-IRF-3 (P-IRF-3). We also investigated the effects of Poly I:C or transfected Poly I:C on the regulation of IFN- $\beta$ production in HCC cells. However, P-IRF-3 was not observed by treatment with either Poly I:C and transfected 
A

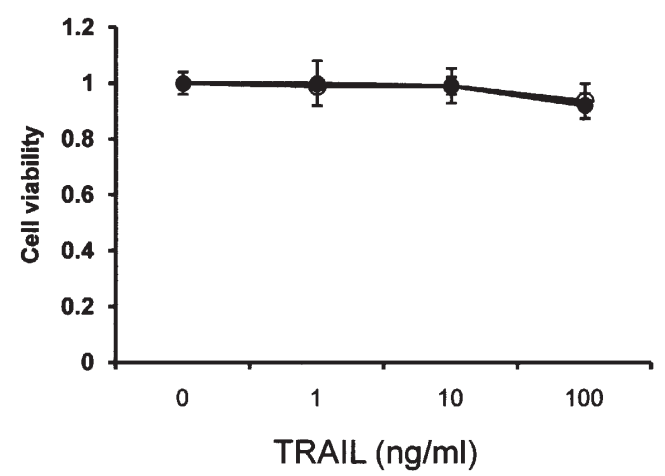

B

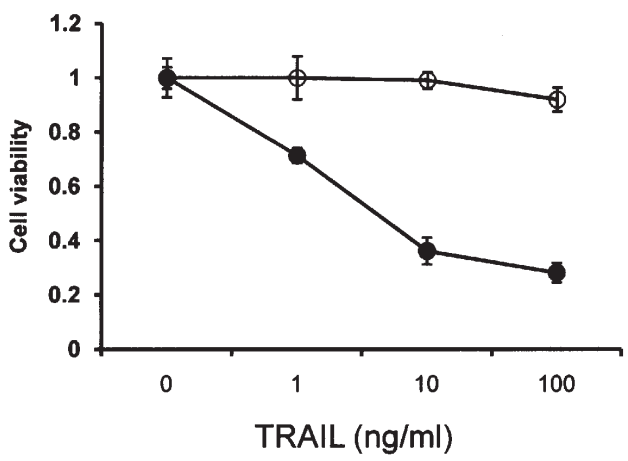

C

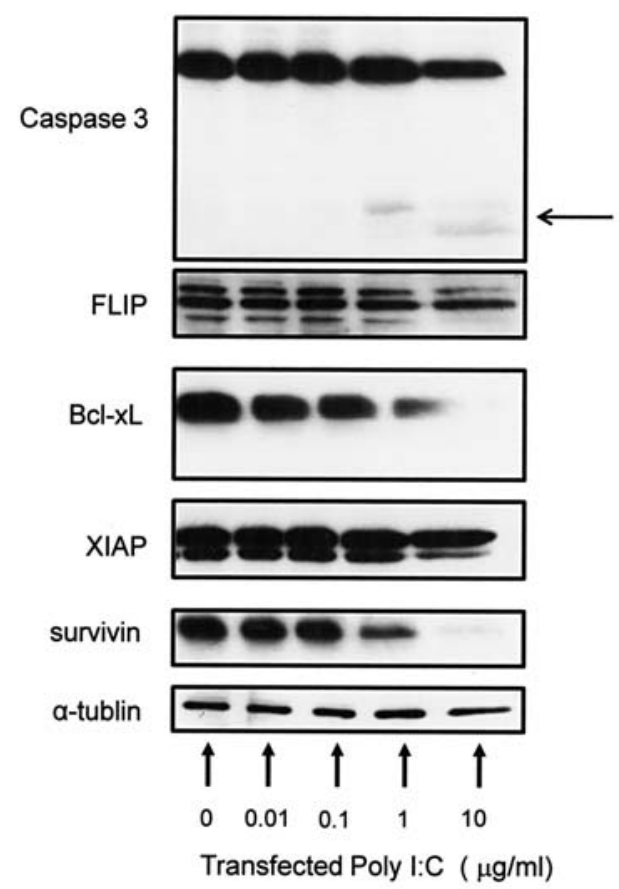

Figure 5. Influence of Poly I:C or transfected Poly I:C on TRAIL-induced apoptosis in HCC cells (Huh7). (A) HCC cells were incubated with various concentrations of TRAIL for $24 \mathrm{~h}$ in the presence (open circle) or absence (closed circle) of $1 \mu \mathrm{g} / \mathrm{ml}$ Poly I:C. (B) HCC cells were incubated with various concentrations of TRAIL for $24 \mathrm{~h}$ in the presence (closed circle) or absence (open circle) of $1 \mu \mathrm{g} / \mathrm{ml}$ transfected Poly I:C. Data shown are the means \pm SD of six independent experiments. (C) Apoptosis-related proteins in response to various concentrations of transfected Poly I:C in HCC cells (Huh7). Transfected Poly I:C down-regulated apoptosis-related proteins. Arrows indicate cleaved caspase 3. Cell lysates were analyzed by SDSPAGE and immunoblotting.

Poly I:C in HCC cells (Fig. 6A). In addition, the amount of IFN-B production was not increased by stimulation with Poly I:C or by transfection with Poly I:C (Fig. 6B).
A

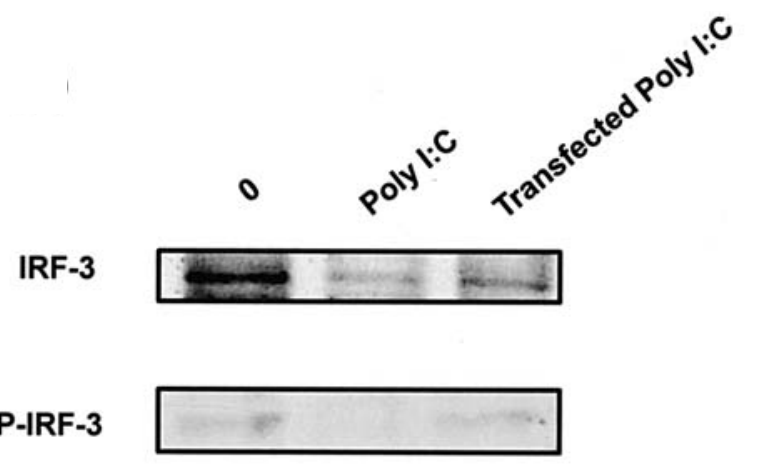

B

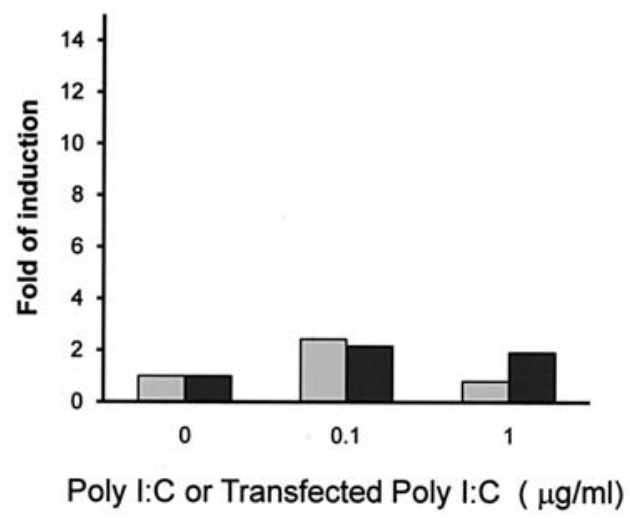

Figure 6. (A) Induction of IRF-3 and P-IRF-3 by Poly I:C and transfected Poly I:C in HCC cells. Cells were incubated with $1 \mu \mathrm{g} / \mathrm{ml}$ Poly I:C, or $1 \mu \mathrm{g} /$ $\mathrm{ml}$ transfected Poly I:C for $24 \mathrm{~h}$. Cell lysates were analyzed by SDS-PAGE and immunoblotting. (B) Effects of IFN- $\beta$ production in Huh7 cells. Cells were treated with Poly I:C or transfected Poly I:C. Concentrations of IFN- $\beta$ in medium were measured by ELISA. The experiment was performed twice with similar results.

\section{Discussion}

In the present study, we used DNA chip analysis to demonstrate for the first time that several TLRs, including TLR3, are expressed in human HCC tissues. TLRs are known to be expressed by various immune cells, including dendritic cells, macrophages and lymphocytes, as well as by non-immune cells such as fibroblasts and epithelial cells (1-4,25-30). Moreover, mRNA coding has been detected in human and murine tumor cell lines, and this expression seems to be conserved in the corresponding transformed cells (20-22,28,31). Since most HCCs develop in HCV infections, we decided to focus on TLR3, as the replicated form of HCV can be recognized by TLR3 (16-19,32-34). The immunohistochemical study revealed that, in addition to non-tumor tissues such as cirrhotic or normal tissues, $52.7 \%$ of the HCC tissues expressed TLR3. We also detected the TLR3 protein in a panel of HCC cell lines. Thus, our results indicate that there was a high prevalence of TLR3 in human HCC. Although some cancer cells, such as colonic adenocarcinoma, lung cancer, breast cancer and melanoma reportedly express TLR3, the exact nature of the expression and the function of TLR3 in cancer cells have yet to be elucidated $(20,21,30,31)$.

Notably, flow cytometric and immune fluorescence staining analyses showed that TLR3 was clearly expressed 
on both the cell surface and in the cytoplasm of HCC cells. TLR1, 2, 4, 5, 6 and 10 are expressed on the cell surface, while TLR3, 7, 8 and 9, which can recognize nucleic acid ligands, are usually expressed in the endosomes and the endoplasmic reticulum $(35,36)$. Unlike immune cells, TLR3 was only detected on the cell surface in fibroblasts and colon epithelial cells, while in melanoma cells, TLR3 was only detectable intracellularly. However, both breast cancer and melanoma cells were able to react with extracellular Poly I:C (21). These results indicate the presence of a cell surface recognition mechanism for TLR3 agonists.

Previous studies suggest that the subcellular localization is of great importance with regard to the discrimination of viral nucleic acids from self nucleic acids $(10,35,36)$. Since currently the exact specifics for the overall mechanism are not known, the significance of the cellular localization of TLR3 remains uncertain. HCC cells are always exposed to various nucleic acids, such as $\mathrm{HBV}$ and $\mathrm{HCV}$ or by the self nucleic acids that are shed due to hepatocyte cell death caused by inflammation. Our findings suggest that HCC cells are able to respond to these various nucleic acids by using both the cell surface and endosomal TLR3.

Therefore, we attempted to elucidate the biological significance of the signaling that occurs via both the cell surface and endosome TLR3. Engagement of the TLR3 signaling pathway leads to the activation of two major transcription factors that have central roles in innate immunity, i.e., NF-кB and IRF-3. Unlike the other TLRs, TLR3 does not recruit the adaptor molecule MyD88, but instead depends solely upon TRIF. Thus, for TLR3, TRIF induces signaling pathways that lead to the activation of NF- $\mathrm{BB}$ and IRF-3 resulting in the initiation of the apoptotic cascade (14,37-39).

In the present study, irrespective of the fact that TLR3 was expressed on the cell surface of HCCs, direct stimulation with Poly I:C did not induce apoptosis. In breast cancer cells, synthetic dsRNA induces apoptosis in a TLR-dependent manner (20). In melanoma cells, TLR3 agonists can directly inhibit cell proliferation and induce tumor cell death (21).

Sub-toxic levels of Act-D and $\mathrm{CHX}$, which inhibit de novo protein synthesis, were able to induce Poly I:C-mediated apoptosis. Signaling components for Poly I:C-induced apoptosis do exist in HCC cells, and thus, our results suggest that a possible endogenous suppressor of Poly I:C-mediated apoptosis might very well exist within HCC cells. In a previous study, we demonstrated that caspase inhibitors, such as survivin, XIAP and FLIP, were sensitive to Act-D and played a critical role in the resistance to apoptosis which occurred via the caspase within these HCC cells (23). Based on these results, we believe that these inhibitors are capable of blocking caspase signaling via cell surface TLR3.

On the other hand, Poly I:C activates NF-кB, particularly in HepG2, Huh7 and SK-Hep1 cells, even though it does not elevate IFN- $\beta$ induction in $\mathrm{HCC}$ cells. NF- $\mathrm{\kappa B}$ usually plays an important role in regulating immune and inflammatory responses, apoptosis and oncogenes (40-42). In cancers, once $\mathrm{NF}-\kappa \mathrm{B}$ is activated, the dimers typically enter the nucleus and encode cytokines, growth factors and anti-apoptotic proteins. Thus, it appears that NF- $\mathrm{\kappa B}$ can convert inflammatory stimuli into tumor growth signals. Thus, HCC cells may use cell surface TLR3 signaling as an anti-apoptosis measure via the activation of $\mathrm{NF}-\kappa \mathrm{B}$, and thus escape immunological cytotoxicity.

Based on our findings, we attempted to stimulate intracellular TLR3 using transfection with Poly I:C. We found that transfected Poly I:C caused dose-dependent apoptotic cell death in all HCC cells. Furthermore, without causing IFN-B production, transfected Poly I:C was able to synergistically augment the TRAIL-induced apoptosis that accompanies the down-regulation of anti-apoptotic proteins, such as Bcl-xL and survivin. These effects were not observed when there was Poly I:C cell surface stimulation.

In conclusion, functional TLR3 is expressed on both the cell surface and in the cytoplasm of HCC cells. Intracellular TLR3 signaling is involved in cell death, while in contrast, the cell surface TLR3 signaling is responsible for activation of NF-кB. The current findings might help to define the clinical perspectives for employing intracellular TLR3 agonists which can be used to directly control cell survival and death. Further evaluation of the possible roles and the type of regulation associated with TLR3 needs to be undertaken.

\section{References}

1. Takeda K, Kaisho T and Akira S: Toll-like receptors. Annu Rev Immunol 21: 335-376, 2003.

2. Takeda $\mathrm{K}$ and Akira S: Toll-like receptors in innate immunity. Int Immunol 17: 1-14, 2005.

3. Beutler B, Jiang Z, Georgel P, et al: Genetic analysis of host resistance: Toll-like receptor signaling and immunity at large. Annu Rev Immunol 24: 353-389, 2006.

4. Kopp E and Medzhitov R: Recognition of microbial infection by Toll-like receptors. Curr Opin Immunol 15: 396-401, 2003.

5. Akira S, Uematsu S and Takeuchi O: Pathogen recognition and innate immunity. Cell 124: 783-801, 2006.

6. Becker MN, Diamond G, Verghese MW and Randell SH: CD14-dependent lipopolysaccharide-induced beta-defensin-2 expression in human tracheobronchial epithelium. J Biol Chem 275: 29731-29736, 2000.

7. Muzio M, Bosisio D, Polentarutti N, et al: Differential expression and regulation of toll-like receptors (TLR) in human leukocytes: selective expression of TLR3 in dendritic cells. J Immunol 164: 5998-6004, 2000.

8. Faure E, Thomas L, Xu H, Medvedev A, Equils O and Arditi M: Bacterial lipopolysaccharide and IFN-gamma induce Toll-like receptor 2 and Toll-like receptor 4 expression in human endothelial cells: role of NF-kappa B activation. J Immunol 166: 2018-2024, 2001.

9. Visintin A, Mazzoni A, Spitzer JH, Wyllie DH, Dower SK and Segal DM: Regulation of Toll-like receptors in human monocytes and dendritic cells. J Immunol 166: 249-255, 2001.

10. Barton GM, Kagan JC and Medzhitov R: Intracellular localization of Toll-like receptor 9 prevents recognition of self-DNA but facilitates access to viral DNA. Nat Immunol 7: 49-56, 2006.

11. Matsumura T, Degawa T, Takii T, et al: TRAF6-NF-kB pathway is essential for interleukin-1-induced TLR2 expression and its functional response to TLR2 ligand in murine hepatocytes. Immunology 109: 127-136, 2003.

12. Harada K, Ohira S, Ise K, et al: Lipopolysaccharide activates nuclear factor $-\kappa \mathrm{B}$ through toll-like receptors and related molecules in cultured biliary epithelial cells. Lab Invest 83: 1657-1667, 2003.

13. Uhrig A, Banafsche R, Kremer M, et al: Development and functional consequences of LPS tolerance in sinusoidal endothelial cells of the liver. J Leukoc Biol 77: 626-633, 2005.

14. Yamamoto M, Sato S, Hemmi H, et al: Role of adaptor TRIF in the MyD88-independent toll-like receptor signaling pathway. Science 301: 640-643, 2003

15. Alexopoulou L, Holt AC, Medzhitov R and Flavell RA: Recognition of double-stranded RNA and activation of NFkappaB by Toll-like receptor 3. Nature 413: 732-738, 2001.

16. Heylbroeck C, Balachandran S, Servant MJ, et al: The IRF-3 transcription factor mediates Sendai virus-induced apoptosis. J Virol 74: 3781-3792, 2001. 
17. Li K, Foy E, Ferreon JC, et al: Immune evasion by hepatitis C virus NS3/4A protease-mediated cleavage of the Toll-like receptor 3 adaptor protein TRIF. Proc Natl Acad Sci USA 102: 2992-2997, 2005

18. Otsuka M, Kato N, Moriyama M, et al: Interaction between the HCV NS3 protein and the host TBK1 protein leads to inhibition of cellular antiviral responses. Hepatology 41: 1004-1012, 2005.

19. Gale M Jr and Foy EM: Evasion of intracellular host defence by hepatitis C virus. Nature 436: 939-945, 2005.

20. Ferreon JC, Ferreon AC, Li K and Lemon SM: Molecular determinants of TRIF proteolysis mediated by the hepatitis $\mathrm{C}$ virus NS3/4A protease. J Biol Chem 280: 20483-20492, 2005.

21. Salaun B, Coste I, Rissoan MC, Lebecque SJ and Renno T: TLR3 can directly trigger apoptosis in human cancer cells. J Immunol 176: 4894-4901, 2006.

22. Salaun B, Lebecque S, Matikainen S, Rimoldi D and Romero P: Toll-like receptor 3 expressed by melanoma cells as a target for therapy? Clin Cancer Res 13: 4565-4574, 2007.

23. Harmey JH, Bucana CD, Lu W, et al: Lipopolysaccharideinduced metastatic growth is associated with increased angiogenesis, vascular permeability and tumor cell invasion. Int $J$ Cancer 101: 415-422, 2002.

24. Yamanaka T, Shiraki K, Sugimoto K, et al: Chemotherapeutic agents augment TRAIL-induced apoptosis in human hepatocellular carcinoma cell lines. Hepatology 32: 482-490, 2000.

25. Matsumoto M, Kikkawa S, Kohase M, Miyake K and Seya T: Establishment of a monoclonal antibody against human Tolllike receptor 3 that blocks double-stranded RNA-mediated signaling. Biochem Biophys Res Commun 293: 1364-1369, 2002.

26. Young SL, Lyddon TD, Jorgenson RL and Misfeldt ML: Expression of Toll-like receptors in human endometrial epithelial cells and cell lines. Am J Reprod Immunol 52: 67-73, 2004.

27. Harada $\mathrm{K}$, Sato $\mathrm{Y}$, Itatsu $\mathrm{K}$, et al: Innate immune response to double-stranded RNA in biliary epithelial cells is associated with the pathogenesis of biliary atresia. Hepatology 46: 1146-1154, 2007.

28. Kumar A, Zhang J and Yu FS: Toll-like receptor 3 agonist poly(I:C)-induced antiviral response in human corneal epithelial cells. Immunology 117: 11-21, 2006.

29. Park C, Lee S, Cho IH, et al: TLR3-mediated signal induces proinflammatory cytokine and chemokine gene expression in astrocytes: differential signaling mechanisms of TLR3-induced IP-10 and IL-8 gene expression. Glia 53: 248-256, 2006.

30. Furrie E, Macfarlane S, Thomson G, Macfarlane GT, Microbiology \& Gut Biology Group; Tayside Tissue \& Tumour Bank: Toll-like receptors-2, -3 and -4 expression patterns on human colon and their regulation by mucosal-associated bacteria. Immunology 115: 565-574, 2005.
31. Uno T, Hirabayashi K, Murai M, Yano J and Ozato K: The role of IFN regulatory factor-3 in the cytotoxic activity of NS-9, a polyinosinic-polycytidylic acid/cationic liposome complex, against tumor cells. Mol Cancer Ther 4: 799-805, 2005.

32. Sumpter R Jr, Loo YM, Foy E, et al: Regulating intracellular anti-viral defense and permissiveness to hepatitis C virus RNA replication through a cellular RNA helicase, RIG-I. J Virol 79: 2689-2699, 2005

33. Yoneyama M, Kikuchi M, Natsukawa T, et al: The RNA helicase RIG-I has an essential function in double-stranded RNAinduced innate antiviral responses. Nat Immunol 5: 730-737, 2004.

34. Li K, Chen Z, Kato N, Gale M Jr and Lemon SM: Distinct polyI:C and virus-activated signaling pathways leading to interferon$\beta$ production in hepatocytes. J Biol Chem 280: 16739-16747, 2005.

35. de Bouteiller O, Merck E, Hasan UA, et al: Recognition of double-stranded RNA by human toll-like receptor 3 and downstream receptor signaling requires multimerization and an acidic pH. J Biol Chem 280: 38133-38145, 2005.

36. Johnsen IB, Nguyen TT, Ringdal M, et al: Toll-like receptor 3 associates with c-Src tyrosine kinase on endosomes to initiate antiviral signaling. EMBO J 25: 3335-3346, 2006.

37. Sharma S, ten Oever BR, Grandvaux N, Zhou GP, Lin R and Hiscott J: Triggering the interferon antiviral response through an IKK-related pathway. Science 300: 1148-1151, 2003.

38. Yang H, Ma G, Lin CH, Orr M and Wathelet MG: Mechanism for transcriptional synergy between interferon regulatory factor. (IRF)-3 and IRF-7 in activation of the interferon-beta gene promoter. Eur J Biochem 271: 3693-3703, 2004.

39. Han KJ, Su X, Xu LG, Bin LH, Zhang J and Shu HB: Mechanisms of the TRIF-induced interferon-stimulated response element and NF-kappaB activation and apoptosis pathways. J Biol Chem 279: 15652-15661, 2004.

40. Baldwin AS Jr: Series introduction: The transcription factor NF-кB and human disease. J Clin Invest 107: 3-6, 2001.

41. Karin M, Cao Y, Greten FR and Li ZW: NF-kappaB in cancer: from innocent bystander to major culprit. Nat Rev Cancer 2 301-310, 2002

42. Ghosh S and Karin M: Missing pieces in the NF-кB puzzle. Cell 109: S81-S96, 2002 